\title{
Detection and Segmentation of Brain Tumor by Thresholding and Bounding-Box using K-Means as a Seed
}

\author{
Chaitra G. \\ Department of DECS \\ VTU-CPGS, VIAT Chickballapur-562101, \\ India
}

\author{
Sarika Tale, $\mathrm{PhD}$ \\ Department of DECS \\ VTU-CPGS, VIAT \\ Chickballapur-562101, India
}

\begin{abstract}
Brain tumor is a mass of abnormal cells replicating in an uncontrolled manner. It affects the growth and function of normal cells in brain and occupies the space in brain. It causes interruption of brain cell function and cause damage to life. Accurate detection and segmentation of brain tumor is challenging task due to several reasons like complex brain structure, increasing data flow, inhomogeneity etc. This paper presents a novel method to segment brain tumor in T1weighted MRI images by employing K-means followed by a thresholding technique and bounding box method by combing these different techniques accuracy of detecting the tumor portion can be increased and finally all the features of the detected tumor such as centroid, solidity, perimeter, area and segmented area and extent are extracted.
\end{abstract}

\section{General Terms}

Clustering, Segmentation, Detection.

\section{Keywords}

K-means technique, Bounding-box, Thresholding, MRI brain images, Fuzzy c-means method.

\section{INTRODUCTION}

The smallest important basic part of all living entities is cell. Humanoid body comprises around millions of cells. Every cell has its individual characteristics and function. These cells split to form additional cells in a well-behaved way for proper functioning of body. When cells split and nurture wildly to form fresh cells, outcomes in a bulk of surplus tissue which is well-defined as a tumor. Wild tissue decay in some portion of the body is named cancer. There are diverse sorts of tumor with dissimilar features and dissimilar cure. Brain tumor occurs as a mass abnormal cells replicating in an uncontrolled manner. It affects the growth and function of normal cells in brain and occupies the space in brain. It causes interruption of brain cell function and cause damage to life [1].

Different techniques are used to analyze the characteristics of brain. Among all MRI is one of the typical modality used in treatment for analysis of brain and cure. This MRI scan provides the complete visualization of anatomy of internal brain tissues which is used in entire process for segmenting brain cancer. The life time of patients suffering from brain tumor can be improved if tumor is identified at earlier phase. MRI scanned images are used for treating the brain tumor and to check any abnormality found on the human brain. To observe and estimate cerebral tumors MRI technique is very suitable because, of its ability to identify difference in compactness of lenient tissues. So, MRI brain images are chosen as input image .In order to get most accurate diagnosis the patient has to undergo several tests to identify the presence of tumor. This will help to give most advanced treatment for the presence of tumor in patient body with minimum impact on patient's body. In health care services, individual risk assessment and medical diagnosis of patients are increasing day by day. In order to cure and prevent the disease the given data of the image is used completely in an effective manner. Nowadays brain cancer cause is increasing day by day, from younger generation people to adult peoples. National Cancer Institute statistics (NCIS) report states that the occurrence of brain tumor is increasing every year by $10 \%$. Combining imaging technology and intelligence software to detect cancer in early stage of development is a challenging task. Diagnosis of cancer patients with tumor and without tumor is one of demanding subject area. Differentiating the occurrence of brain tumor in brain MR image of cancer patients, and healthy people to detect and segment tumor is necessary [2].

MRI brain images are developed by diverse procedures, e.g. T1 biased images, T1 weighted images-Tumor portion is found with high intensity in contrast with $\mathrm{T} 2$ weighted images. In T1 weighted images the fat tissues appear to be bright and CSF as black. In T2 images the fat tissues appears to be dark and CSF as bright. Dissimilar brain tissues resemble to dissimilar tissue categories, which are existing in the brain. Combination of brain into three key brain tissue classes (Cerebrospinal fluid, Gray Matter, White Matter) is considered very significant in expectancy and cure of several diseases [3].

\section{RELATED WORK}

Authors show that segmentation of brain tumor can be achieved by watershed segmentation by grouping pixels of an image based on their intensity values but, suffers from over segmentation problem due, to presence of noise or variation of intensity [4].

In case of the region growing based segmentation user has to select the seed. Hence it needs more interaction of user in selection of seed. Seed is center point of the tumor cells.Region growing method may cause intensity in homogeneity problem. And it lacks to give aacurate results for all the images[5].

Edge detection will give exact and clear boundaries or edges. But its disadvantage is that if the intensity difference between normal and tumor cells is less, it will not be detected. But it will give exact size of tumor when detected.The edge detection technique work well on high contrast images and itlacks to detect the edges in low contrast noisy images due to the weak gradient magnitude [6].

Fuzzy c means is also another approach used used segmentation and detetion of abnornal tissues in slice of MRI image [7]. This method involves many mathematical operations and formulas, hence has a high computation 
complexity like mean shift algorithm, FCM is suitable when the number of clusters is unknown a priori [8].

In marker controlled watershed segmentation the internal and external markers required for segmentation purpose are computed based on connected components of pixels. Internal markers are the connected components of pixels associated with objects of interest.The external markers are the connected components of pixels associated with the background of the objects. These markers are used for segmentation purpose to extract the tumor portion. In marker based segmentation algorithm selecting markers manually is very crucial hence automatic selection of marker is required [9].

\section{METHODOLOGY}

Proposed method includes following steps in accurate detection and segmentation of brain tumor tissues

- Database acquisition

- K-means method

- Thresholding technique

- Bounding box method

- Feature extraction

\subsection{Image acquisition}

The image acquisition involves downloading and storing T1 weighted MR brain images database from publically available online source. MR brain images of different age group with different planes of brain such as sagital, coronal, and axial are collected and stored.

\subsection{K-means method}

The MRI image is set has input to $\mathrm{k}$-means algorithm.It groups the pixels into different clusters based on their intensities values. It select the centers of clusters or seed points randomly and starts calculating the distance between the pixel in image with all clusters using euclidian distance formula taking the intensity values of seed point and pixel. If the distance is close to cluster then move the pixel to respective cluster. This is done for predetermined levels. After grouping the pixels a mean value is again calculated if the distance of pixel is close to mean value then it is retained in same cluster or else moved to other cluster . like this a set of iterations is followed untill center converges .The pixels in one paricular cluster should have a minimum ditance to its mean value. If not the pixel is moved to another cluster whose mean value is close to that pixel [10].

\subsection{Thresholding technique}

Segmentation of tumor using thresholding procedure is one of simplest technique to extract tumor portion. K-means method is followed by threshold segmentation converts input gray scale image to binary format based on threshold range. The optimal threshold range is selected from image to divide pixels in different classes and distinguish the object from background these pixels are divided based on their intensity values using histogram for segmentation purpose. The thresholding technique is applied to the segmented tumor extracted from the brain in order to eliminate the healthy tissues misclassified with tumor region. The thresholding technique is applied in this proposed system is optimal threshold technique applied for each image individually because choosing threshold value for each image is more complex. The threshold value is keep on increasing for segmented tumor in order to obtain the fine tumor region [11].

\subsection{Bounding-box method}

The segmented tumor portion from K-means and threshold technique is superimposed with by bounding box approach. This approach places the bounding box around the tumor portion in segmented tumor of MRI. The segmented tumor from the K-means method contains not only the tumor portion but also some healthy tissues will be misclassified. These cells are eliminated by applying the threshold technique that is increasing the threshold value until the misclassified tissues are eliminated to obtain fine tumor. Then finally bounding box will be placed around the tumor region based on the shape attributes of the detected tumor. The features from the detected tumor is extracted such as centroid, area, perimeter, solidity, extent and segmented area [12]

\subsection{Feature extraction}

Feature extraction is done for the detected and segmented tumor based on the shape attributes of the tumor. The features from the detected tumor are extracted such as centroid, area, perimeter, solidity, extent and segmented area and in future it is used for classification purpose.

\section{RESULTS}

MRI image is given as input and after applying k-means algorithm affected tumor is segmented out and displayed on graphical user interface in figure 1 The tumor portion extracted from MRI brain image contains healthy cells which are misclassified. Along with the tumor portion some other intensity values which are loosely packed are also present hence in order to eliminate set.

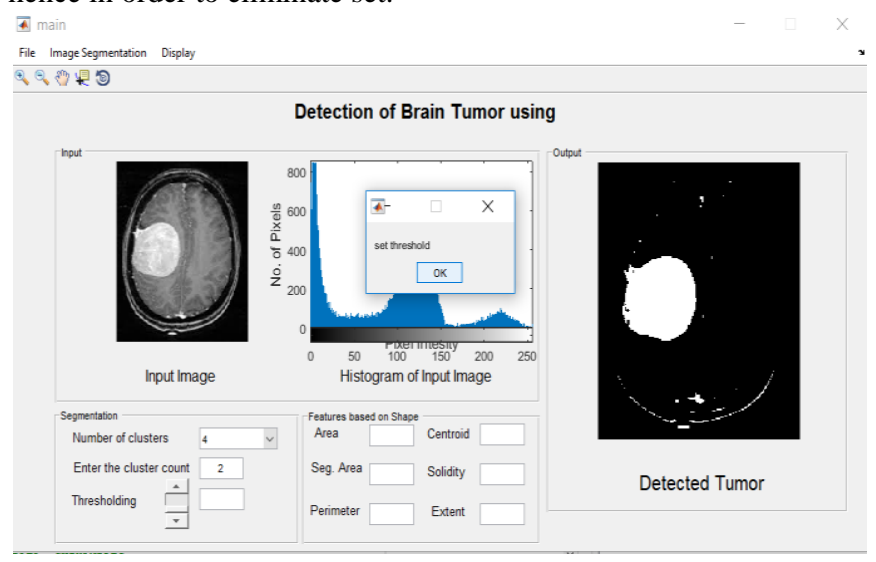

Fig 1: Segmented tumor by K-means

Threshold value set to 60 in figure 2 below which all the misclassified cells are eliminated leaving behind only fine portion of tumor.

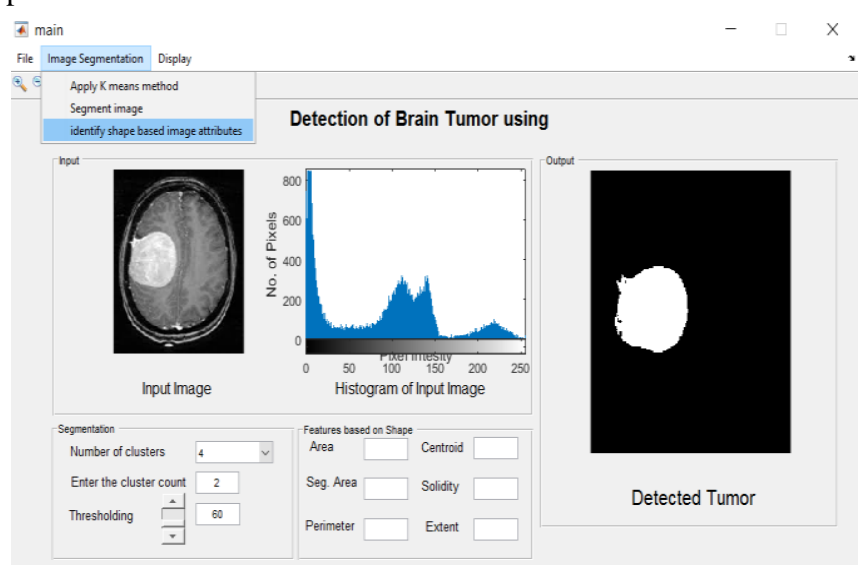

Fig 2: Fine tumor by threshold 
Final output of the detected tumor superimposed on original image by bounding box around the tumor region in Figure 3 The features of detected tumor such as area, segmented area, perimeter, centroid, solidity, extent are also calculated.

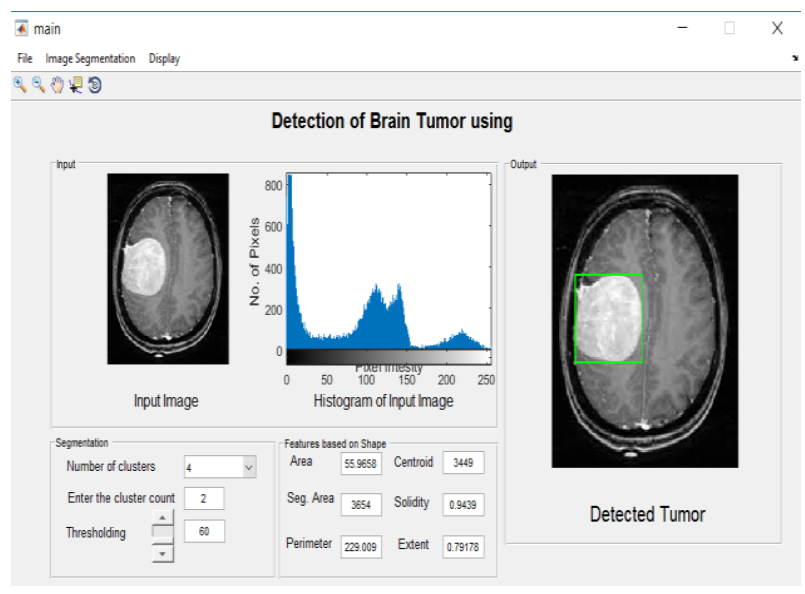

Fig 3: Detected tumor

\section{CONCLUSION}

Clustering method alone cannot provide accurate results because in segmenting the tumor by k-means along with tumor portion some of the healthy tissues may also be misclassified hence in order to avoid this. Clustering method followed by threshold technique to reject extra tissues in demand to sense merely which matching tumor. Then finally result is evaluated with bounding box method. The experimental outcomes have revealed this method very strong in spotting or bounding affected tumor cells of MR images in spite of the inhomogeneity problem and also intensity variation or irregular structure of tumor. Distinct one-phase method, projected method is vigorous to outline or structure variation of tumor also no dataset is required for training. Future work can be extended to classify the tumor based on the features extracted from the detected tumor and this technique can be applied in 3-D images in future to segment and detect the tumor portion

\section{ACKNOWLEDGMENTS}

I would like to express my special thanks of gratitude to prof. Dr. Sarika Tale (HOD), Department of Digital Electronic and Communication Systems, Visvesvaraya Institute of Advanced Technology. Who gave me the golden opportunity to do this wonderful project on the topic (Detection and Segmentation of Brain Tumor by Thresholding and Bounding-box using Kmeans as a Seed), which also helped me in doing a lot of research and I came to know about so many new things we are really thankful to them. And, secondly I would also like to thank my parents and friends who helped me a lot in finalizing this project within the limited time frame.

\section{REFERENCES}

[1] S. Aswathy, G. Glan Deva Dhas, and S. Kumar A survey on detection of brain tumor from MRI brain images," in Control, Instrumentation, Communication and Computational Technologies (ICCICCT), 2014 International Conference on, July 2014, pp. 871-877.
[2] S.Bauer, R. Wiest, L.-P. Nolte, and M. Reyes, "A survey of MRI-based medical image analysis for brain tumor studies," Physics in Medicine and Biology, vol. 58, no. 13, pp. $97-129,2013$.

[3] Swapnil R. Telrandhe1 Amit Pimpalkar Ankita Kendhe "Detection of Brain Tumor from MRI images by using Segmentation" 2016 World Conference on Futuristic Trends in Research and Innovation for Social Welfare (WCFTR'16)

[4] Pooja Thakur, Dr. Kuldip Pahwa and Dr. Rajat Gupta ]"Brain Tumor Detection, Segmentation Using Watershed Segmentation And Morphological operation" international Journal of Computer Applications (0975 8887) Volume 58- No.16, November 2012

[5] S.Charutha and M. Jayashree, "An efficient brain tumor detection by texture based region growing and cellular automata edge detection," in Control, Instrumentation, Communication and Computational Technologies (ICCICCT), 2014 International Conference on, July 2014, pp. 1193-1199.

[6] Pratibha Sharma ,Manoj Diwakar ,Sangam Choudhary "Application of Edge Detection for Brain Tumor Detection" International Journal of Advanced Research in Electronics and Communication Engineering (IJARECE) Volume4, Issue 6, June 2015

[7] R. Preetha and G. Suresh, "Performance analysis of fuzzy c means algorithm in automated detection of brain tumor," in Computing and Communication Technologies (WCCCT), 2014 World Congress on, Feb 2014, pp.3033.

[8] Alan Jose1, S. Ravi, M. Sambath "Brain Tumor Segmentation Using K-Means Clustering And Fuzzy CMeans Algorithms And Its Area Calculation" International Journal of Innovative Research in Computer and Communication Engineering. Vol. 2, Issue 3, March 2014

[9] Benson C. C.1, Lajish V. L, Kumar Rajamani "Brain Tumor Extraction from MRI Brain Images Using Marker Based Watershed Algorithm"3189 78-1-4799-87924/15/\$31.00 c 2015 IEEE.

[10] J. Vijay and J. Subhashini, "An efficient brain tumor detection methodology using k-means clustering algorithm in Communications and Signal Processing (ICCSP), 2013 International Conference on, April 2013, pp. 653-657.

[11] Natarajan P and sushmitha G ]"Brain tumor detection using threshold operation" international journal of pharmacy and technology

[12] T. E. M. A. Bianchi A, Miller JV, "Brain tumor segmentation with symmetric texture and symmetric intensity-based decision forests," in Proceedings / IEEE International Symposium on Biomedical Imaging: from nano to macro IEEE International Symposium on Biomedical Imaging., 2013, pp. 748 - 751 . 